\title{
Dipolar droplet bound in a trapped Bose-Einstein condensate
}

\author{
Luis E. Young-S.* and S. K. Adhikari ${ }^{\dagger}$ \\ Instituto de Física Teórica, UNESP-Universidade Estadual Paulista, 01.140-070 São Paulo, São Paulo, Brazil
}

(Received 9 November 2012; published 17 January 2013)

\begin{abstract}
We study the statics and dynamics of a dipolar Bose-Einstein condensate (BEC) droplet bound by interspecies contact interaction in a trapped nondipolar BEC. Our findings are demonstrated in terms of stability plots of a dipolar ${ }^{164} \mathrm{Dy}$ droplet bound in a trapped nondipolar ${ }^{87} \mathrm{Rb} \mathrm{BEC}$ with a variable number of ${ }^{164} \mathrm{Dy}$ atoms and interspecies scattering length. A trapped nondipolar BEC of a fixed number of atoms can bind only a dipolar droplet containing fewer atoms than a critical number for the interspecies scattering length between two critical values. The shape and size (statics) as well as the small breathing oscillation (dynamics) of the dipolar BEC droplet are studied using numerical and variational solutions of a mean-field model. We also suggest an experimental procedure for achieving such a ${ }^{164} \mathrm{Dy}$ droplet by relaxing the trap on the ${ }^{164} \mathrm{Dy} \mathrm{BEC}$ in a trapped binary ${ }^{87} \mathrm{Rb}-{ }^{164} \mathrm{Dy}$ mixture.
\end{abstract}

DOI: 10.1103/PhysRevA.87.013618

PACS number(s): 03.75.Hh, 03.75.Mn, 03.75.Kk

\section{INTRODUCTION}

The experimental observation of dipolar Bose-Einstein condensates (BECs) of ${ }^{52} \mathrm{Cr}$ [1-5], ${ }^{164}$ Dy [6,7], and ${ }^{168} \mathrm{Er}[8]$ atoms with large magnetic dipole moments has opened new directions of research in cold atoms in the quest of novel and interesting features related to the anisotropic long-range dipolar interaction. Polar molecules, with much larger (electric) dipole moment, are also being considered [9] for BEC experiments. The atomic interaction in a dilute BEC of alkali metal and other types of atoms (with negligible dipole moment) is represented by an $S$-wave contact ( $\delta$-function) potential. However, the nonlocal anisotropic long-range dipolar interaction acts in all partial waves and is attractive in certain directions and repulsive in others.

An untrapped three-dimensional (3D) BEC with attractive interaction does not exist in nature due to collapse instability [10]. However, the collapse of an untrapped BEC can be avoided due to the interspecies contact interaction in a binary mixture with a trapped BEC. The shape of such a stable droplet bound in a trapped BEC is controlled by the interspecies interactions, whereas that of a trapped BEC is determined by the underlying trap. Here we consider such a dipolar droplet bound in a trapped nondipolar BEC. The effect of the underlying atomic interaction, especially that of the anisotropic dipolar interaction, will easily manifest itself in such a dipolar droplet. These droplets will be termed quasifree as they can easily move around inside the larger trapped BEC responsible for their binding. By taking the trapped BEC to be nondipolar, one can easily study the effect of intraspecies dipolar interaction on the quasifree droplet in the absence of any interspecies dipolar interaction.

Dipolar BECs are immediately distinguishable from those with purely contact interactions by their strong shape and stability dependence [11] on the trapping geometry. Here, we are proposing a different way to trap a dipolar BEC in the form of a quasifree dipolar droplet, using an attractive interspecies mean-field potential, which introduces a unique

\footnotetext{
*lyoung@ift.unesp.br

†adhikari@ift.unesp.br; URL:http://www.ift.unesp.br/users/adhikari
}

trapping geometry that results in further interesting shape and stability characteristics of dipolar BECs. We study the statics and dynamics of a quasifree dipolar droplet using numerical solution and variational approximation of a meanfield model [12,13]. For this purpose we consider a binary mixture of nondipolar ${ }^{87} \mathrm{Rb}$ and dipolar ${ }^{164} \mathrm{Dy}$ where the trapped nondipolar ${ }^{87} \mathrm{Rb} \mathrm{BEC}$ could be in a cigar or disk shape. Among the available dipolar BECs $[4,7,8],{ }^{164}$ Dy atoms have the largest (magnetic) dipolar interaction strength [1,6]. The existence of stable 3D ${ }^{164}$ Dy droplets is illustrated by stability plots involving the number of atoms of the two species and the interspecies scattering length. For a fixed number $N(\mathrm{Rb})$ of ${ }^{87} \mathrm{Rb}$ atoms the dipolar droplet could be bound below a critical number $N$ (Dy) of ${ }^{164}$ Dy atoms between two limiting values of interspecies attraction. If the interspecies attraction is too small, the ${ }^{164}$ Dy atoms in the droplet cannot be bound and, for a very large interspecies attraction, the droplet is destroyed by collapse instability. Usually, the dipolar droplet has the same shape as the trap acting on the nondipolar BEC. However, for a large number of ${ }^{164} \mathrm{Dy}$ atoms, as the interspecies attraction approaches the collapse instability, the dipolar droplet always is of cigar shape even if the external trap is disk shaped, and eventually the dipolar droplet collapses on the axial $z$ axis from the cylindrical configuration. For a small number of atoms, the dipolar droplet collapses to the center, maintaining its shape, rather than on the axial $z$ axis.

The variational approximation to the sizes and chemical potentials of the stationary droplets is compared with the numerical solution of the mean-field model. The numerical study of breathing oscillation of the stable dipolar droplet is found to be in reasonable agreement with a time-dependent variational model calculation. We also demonstrate a viable experimental way of creating a ${ }^{164}$ Dy droplet bound in a trapped ${ }^{87} \mathrm{Rb} \mathrm{BEC}$, e.g., by slowly removing the trap on a trapped binary ${ }^{164} \mathrm{Dy}-{ }^{87} \mathrm{Rb}$ mixture, while the trapped ${ }^{164} \mathrm{Dy}$ BEC evolves into a quasifree droplet.

In Sec. II the mean-field model for a dipolar BEC droplet bound in a trapped nondipolar BEC is developed. A timedependent, analytic, Euler-Lagrange Gaussian variational approximation of the model is also presented. The results of numerical calculation are shown in Sec. III. Finally, in Sec. IV we present a brief summary of our findings. 


\section{MEAN-FIELD MODEL FOR A QUASIFREE DIPOLAR DROPLET}

We consider a binary BEC, where one of the species is dipolar and the other nondipolar, interacting via inter- and intraspecies interactions with the mass, number, magnetic dipole moment, and scattering length for the two species $i=1,2$, denoted by $m_{i}, N_{i}, \mu_{i}$, and $a_{i}$, respectively. The first species $\left({ }^{87} \mathrm{Rb}\right)$ is taken to be nondipolar $\left(\mu_{1}=0\right)$ and trapped while the second species $\left({ }^{164} \mathrm{Dy}\right)$ is dipolar $\left(\mu_{2} \neq 0\right)$ and polarized along the axial $z$ direction. The angular frequencies for the axially symmetric trap on ${ }^{87} \mathrm{Rb}$ along the $x, y$, and $z$ directions are taken as $\omega_{x}=\omega_{y}=\omega_{1}$ and $\omega_{z}=\lambda_{1} \omega_{1}$. The inter- $\left(V_{12}\right)$ and intra- $\left(V_{i}\right)$ species interactions for two atoms at positions $\mathbf{r}$ and $\mathbf{r}^{\prime}$ are taken as

$$
\begin{gathered}
V_{12}(\mathbf{R})=\frac{2 \pi \hbar^{2} a_{12}}{m_{R}} \delta(\mathbf{R}), \quad V_{1}(\mathbf{R})=\frac{4 \pi \hbar^{2} a_{1}}{m_{1}} \delta(\mathbf{R}), \\
V_{2}(\mathbf{R})=\frac{\mu_{0} \mu_{2}^{2}}{4 \pi} \frac{1-3 \cos ^{2} \theta}{|\mathbf{R}|^{3}}+\frac{4 \pi \hbar^{2} a_{2}}{m_{2}} \delta(\mathbf{R}),
\end{gathered}
$$

where $\mathbf{R}=\mathbf{r}-\mathbf{r}^{\prime}, \mu_{0}$ is the permeability of free space, $\theta$ is the angle made by the vector $\mathbf{R}$ with the polarization $z$ direction, and $m_{R}=m_{1} m_{2} /\left(m_{1}+m_{2}\right)$ is the reduced mass of the two species of atoms. With these interactions, the coupled Gross-Pitaevskii (GP) equations for the binary dipolar BEC can be written as [13]

$$
\begin{aligned}
& i \hbar \frac{\partial \phi_{1}(\mathbf{r}, t)}{\partial t} \\
& =\left[-\frac{\hbar^{2}}{2 m_{1}} \nabla^{2}+\frac{1}{2} m_{1} \omega_{1}^{2}\left(\rho^{2}+\lambda_{1}^{2} z^{2}\right)\right. \\
& \left.\quad+\frac{4 \pi \hbar^{2}}{m_{1}} a_{1} N_{1}\left|\phi_{1}(\mathbf{r}, t)\right|^{2}+\frac{2 \pi \hbar^{2}}{m_{R}} a_{12} N_{2}\left|\phi_{2}(\mathbf{r}, t)\right|^{2}\right] \phi_{1}(\mathbf{r}, t), \\
& \quad+\frac{2 \pi \hbar^{2}}{m_{R}} a_{12} N_{1}\left|\phi_{1}(\mathbf{r}, t)\right|^{2}+N_{2} \frac{\mu_{0} \mu_{2}^{2}}{4 \pi} \\
& \left.\quad \times \int V_{d d}(\mathbf{R})\left|\phi_{2}\left(\mathbf{r}^{\prime}, t\right)\right|^{2} d \mathbf{r}^{\prime}\right] \phi_{2}(\mathbf{r}, t) \\
& \quad\left[-\frac{\hbar^{2}}{2 m_{2}} \nabla^{2}+\frac{4 \pi \hbar^{2}}{m_{2}} a_{2} N_{2}\left|\phi_{2}(\mathbf{r}, t)\right|^{2}\right. \\
& V_{d d}(\mathbf{R})=\frac{1-3)}{\cos ^{2} \theta}, \quad \rho^{2}=x^{2}+y^{2}, \quad i=\sqrt{-1} .
\end{aligned}
$$

To compare the dipolar and contact interactions, the intraspecies dipolar interaction is expressed in terms of the length scale $a_{d d}$, defined by $a_{d d}=\mu_{0} \mu_{2}^{2} m_{2} /\left(12 \pi \hbar^{2}\right)$. We express the strength of the dipolar interaction in Eq. (4) by this length scale and transform Eqs. (3) and (4) into the following dimensionless form [13]:

$$
\begin{aligned}
i \frac{\partial \phi_{1}(\mathbf{r}, t)}{\partial t}= & {\left[-\frac{\nabla^{2}}{2}+\frac{1}{2}\left(\rho^{2}+\lambda_{1}^{2} z^{2}\right)+g_{1}\left|\phi_{1}\right|^{2}\right.} \\
& \left.+g_{12}\left|\phi_{2}\right|^{2}\right] \phi_{1}(\mathbf{r}, t), \\
i \frac{\partial \phi_{2}(\mathbf{r}, t)}{\partial t}=[ & -m_{12} \frac{\nabla^{2}}{2}+g_{2}\left|\phi_{2}\right|^{2}+g_{21}\left|\phi_{1}\right|^{2} \\
& \left.+g_{d d} \int V_{d d}(\mathbf{R})\left|\phi_{2}\left(\mathbf{r}^{\prime}, t\right)\right|^{2} d \mathbf{r}^{\prime}\right] \phi_{2}(\mathbf{r}, t),
\end{aligned}
$$

where $\quad m_{12}=m_{1} / m_{2}, g_{1}=4 \pi a_{1} N_{1}, g_{2}=4 \pi a_{2} N_{2} m_{12}$, $g_{12}=2 \pi m_{1} a_{12} N_{2} / m_{R}, g_{21}=2 \pi m_{1} a_{12} N_{1} / m_{R}$, and $g_{d d}=$ $3 N_{2} a_{d d} m_{12}$. In Eqs. (6) and (7), length is expressed in units of the oscillator length of the first species $l_{0}=\sqrt{\hbar / m_{1} \omega_{1}}$, energy in units of the oscillator energy $\hbar \omega_{1}$, density $\left|\phi_{i}\right|^{2}$ in units of $l_{0}^{-3}$, and time in units of $t_{0}=\omega_{1}^{-1}$.

A convenient analytic variational approximation to Eqs. (6) and (7) can be obtained with the following ansatz for the wave functions [14-16]:

$\phi_{i}(\mathbf{r}, t)=\frac{\pi^{-3 / 4}}{w_{\rho i} \sqrt{w_{z i}}} \exp \left[-\frac{\rho^{2}}{2 w_{\rho i}^{2}}-\frac{z^{2}}{2 w_{z i}^{2}}+i \alpha_{i} \rho^{2}+i \beta_{i} z^{2}\right]$,

where $\mathbf{r}=\{\vec{\rho}, z\}, \vec{\rho}=\{x, y\}, w_{\rho i}$ and $w_{z i}$ are the widths, and $\alpha_{i}$ and $\beta_{i}$ are additional variational parameters. The effective Lagrangian for the binary system is

$$
\begin{aligned}
L= & \int d \mathbf{r} \frac{1}{2}\left[\sum_{i}\left\{i N_{i}\left(\phi_{i} \dot{\phi}_{i}^{*}-\phi_{i}^{*} \dot{\phi}_{i}\right)+N_{i} g_{i}\left|\phi_{i}(\mathbf{r})\right|^{4}\right\}\right. \\
& +N_{1}\left|\nabla \phi_{1}(\mathbf{r})\right|^{2}+m_{12} N_{2}\left|\nabla \phi_{2}(\mathbf{r})\right|^{2}+N_{1}\left(\rho^{2}+\lambda_{1}^{2} z^{2}\right) \\
& \left.\times\left|\phi_{1}(\mathbf{r})\right|^{2}\right]+N_{1} g_{12} \int d \mathbf{r}\left|\phi_{1}(\mathbf{r})\right|^{2}\left|\phi_{2}(\mathbf{r})\right|^{2} \\
& +\frac{N_{2}}{2} g_{d d} \iint V_{d d}(\mathbf{R})\left|\phi_{2}\left(\mathbf{r}^{\prime}\right)\right|^{2}\left|\phi_{2}(\mathbf{r})\right|^{2} d \mathbf{r}^{\prime} d \mathbf{r}, \\
= & \sum_{i=1}^{2} \frac{N_{i}}{2}\left(2 w_{\rho i}^{2} \dot{\alpha}_{i}+w_{z i}^{2} \dot{\beta}_{i}\right)+\frac{N_{1}}{2}\left[\frac{1}{w_{\rho 1}^{2}}+\frac{1}{2 w_{z 1}^{2}}\right. \\
& \left.+4 w_{\rho 1}^{2} \alpha_{1}^{2}+2 w_{z 1}^{2} \beta_{1}^{2}\right]+\frac{N_{2} m_{12}}{2}\left[\frac{1}{w_{\rho 2}^{2}}+\frac{1}{2 w_{z 2}^{2}}\right. \\
& \left.+4 w_{\rho 2}^{2} \alpha_{2}^{2}+2 w_{z 2}^{2} \beta_{2}^{2}\right]+\frac{N_{1}}{2}\left[w_{\rho 1}^{2}+\lambda_{1}^{2} \frac{w_{z 1}^{2}}{2}\right] \\
& +\frac{N_{1}^{2} a_{1}}{\sqrt{2 \pi} w_{\rho 1}^{2} w_{z 1}}+\frac{N_{2}^{2} m_{12}}{\sqrt{2 \pi} w_{\rho 2}^{2} w_{z 2}}\left[a_{2}-a_{d d} f(\kappa)\right] \\
& +\frac{C N_{1} N_{2}}{2 A B^{1 / 2}}, \quad \kappa=\frac{w_{\rho 2}}{w_{z 2}}, \\
f(\kappa)= & \frac{1+2 \kappa^{2}-3 \kappa^{2} d(\kappa)}{1-\kappa^{2}}, d(\kappa)=\frac{\arctan \left(\sqrt{\kappa^{2}-1}\right)}{\sqrt{\kappa^{2}-1}}
\end{aligned}
$$

where $A=w_{\rho 1}^{2}+w_{\rho 2}^{2}, B=w_{z 1}^{2}+w_{z 2}^{2}$, and $C=4 a_{12} m_{1} /$ $\left(\sqrt{\pi} m_{R}\right)$. In these equations the overdot denotes the time derivative. The Euler-Lagrange variational equations for the widths for the effective Lagrangian (10), obtained in the usual fashion [16], are

$$
\begin{gathered}
\ddot{w}_{\rho 1}+w_{\rho 1}=\frac{1}{w_{\rho 1}^{3}}+\frac{1}{\sqrt{2 \pi}} \frac{2 N_{1} a_{1}}{w_{\rho 1}^{3} w_{z 1}}+\frac{C N_{2} w_{\rho 1}}{A^{2} B^{1 / 2}}, \\
\ddot{w}_{z 1}+\lambda_{1}^{2} w_{z 1}=\frac{1}{w_{z 1}^{3}}+\frac{1}{\sqrt{2 \pi}} \frac{2 N_{1} a_{1}}{w_{\rho 1}^{2} w_{z 1}^{2}}+\frac{C N_{2} w_{z 1}}{A B^{3 / 2}}, \\
\ddot{w}_{\rho 2}=\frac{m_{12}}{w_{\rho 2}^{3}}+\frac{N_{2} m_{12}\left[2 a_{2}-a_{d d} g(\kappa)\right]}{\sqrt{2 \pi} w_{\rho 2}^{3} w_{z 2}}+\frac{C N_{1} w_{\rho 2}}{A^{2} B^{1 / 2}}, \\
\ddot{w}_{z 2}=\frac{m_{12}}{w_{z 2}^{3}}+\frac{2 N_{2} m_{12}\left[a_{2}-a_{d d} h(\kappa)\right]}{\sqrt{2 \pi} w_{\rho 2}^{2} w_{z 2}^{2}}+\frac{C N_{1} w_{z 2}}{A B^{3 / 2}},
\end{gathered}
$$




$$
\begin{aligned}
g(\kappa) & =\frac{2-7 \kappa^{2}-4 \kappa^{4}+9 \kappa^{4} d(\kappa)}{\left(1-\kappa^{2}\right)^{2}}, \\
h(\kappa) & =\frac{1+10 \kappa^{2}-2 \kappa^{4}-9 \kappa^{2} d(\kappa)}{\left(1-\kappa^{2}\right)^{2}} .
\end{aligned}
$$

The solution of the time-dependent Eqs. (11)-(14) gives the dynamics of the variational approximation.

If $\mu_{i}$ is the chemical potential with which the stationary wave function $\phi_{i}(\mathbf{r}, t)$ propagates in time, e.g., $\phi_{i}(\mathbf{r}, t) \sim$ $\exp \left(-i \mu_{i} t\right) \phi_{i}(\mathbf{r})$, then the variational estimate for $\mu_{i}$ is

$$
\begin{aligned}
\mu_{1}= & \frac{1}{2}\left[\frac{1}{w_{\rho 1}^{2}}+\frac{1}{2 w_{z 1}^{2}}\right]+\frac{1}{2}\left[w_{\rho 1}^{2}+\lambda_{1}^{2} \frac{w_{z 1}^{2}}{2}\right] \\
& +\frac{2 N_{1} a_{1}}{\sqrt{2 \pi} w_{\rho 1}^{2} w_{z 1}}+\frac{C N_{2}}{2 A B^{1 / 2}}, \\
\mu_{2}= & \frac{m_{12}}{2}\left[\frac{1}{w_{\rho 2}^{2}}+\frac{1}{2 w_{z 2}^{2}}\right] \\
+ & \frac{2 N_{2} m_{12}}{\sqrt{2 \pi} w_{\rho 2}^{2} w_{z 2}}\left[a_{2}-a_{d d} f(\kappa)\right]+\frac{C N_{1}}{2 A B^{1 / 2}} .
\end{aligned}
$$

The total energy of the system is given by

$$
\begin{aligned}
E= & \frac{N_{1}}{2}\left[\frac{1}{w_{\rho 1}^{2}}+\frac{1}{2 w_{z 1}^{2}}\right]+\frac{N_{1}}{2}\left[w_{\rho 1}^{2}+\lambda_{1}^{2} \frac{w_{z 1}^{2}}{2}\right] \\
& +\frac{N_{2} m_{12}}{2}\left[\frac{1}{w_{\rho 2}^{2}}+\frac{1}{2 w_{z 2}^{2}}\right]+\frac{N_{1}^{2} a_{1}}{\sqrt{2 \pi} w_{\rho 1}^{2} w_{z 1}} \\
& +\frac{N_{2}^{2} m_{12}}{\sqrt{2 \pi} w_{\rho 2}^{2} w_{z 2}}\left[a_{2}-a_{d d} f(\kappa)\right]+\frac{C N_{1} N_{2}}{2 A B^{1 / 2}} .
\end{aligned}
$$

The widths of the stationary state can be obtained from the solution of Eqs. (11)-(14) by setting the time derivatives of the widths equal to zero. This procedure is equivalent to a minimization of the energy (19), provided the stationary state is stable and corresponds to an energy minimum.

\section{NUMERICAL RESULTS}

We solve Eqs. (6) and (7) by a split-step Crank-Nicolson discretization scheme using a space step of 0.1 and the time step $0.001[15,17]$. The contribution of the dipolar interaction is calculated in momentum space by Fourier transformation [15]. For both species of atoms ${ }^{87} \mathrm{Rb}$ and ${ }^{164} \mathrm{Dy}$ we take the intraspecies scattering length as $a_{i}=110 a_{0}$, and the strength of dipolar interaction as $a_{d d}=131 a_{0}[7,10]$. The yet unknown interspecies scattering length $a_{12}$ is taken as a variable. The variation of $a_{12}$ can be achieved experimentally by the Feshbach resonance technique [18]. We consider the trap frequency $\omega_{1}=2 \pi \times 115 \mathrm{~Hz}$, so that the length scale $l_{0} \equiv \sqrt{\hbar / m_{1} \omega_{1}}=1 \mu \mathrm{m}$ and time scale $t_{0} \equiv \omega_{1}^{-1}=1.38 \mathrm{~ms}$.

We find that a quasifree ${ }^{164} \mathrm{Dy}$ droplet is achievable for a moderately attractive interspecies attraction (negative $a_{12}$ ) and for appropriate values of the number of atoms of the two species $N_{1}$ and $N_{2}$. We illustrate the domain of existence of a stable ${ }^{164}$ Dy droplet in terms of stability plots in Figs. 1(a), $1(\mathrm{~b})$, and $1(\mathrm{c})$ for $N(\mathrm{Rb})=2000,10000$, and 50000 , and for $\lambda=4$ (disk-shaped trap) and 0.25 (cigar-shaped trap). We consider cigar- and disk-shaped ${ }^{164}$ Dy droplets in this study, where the effect of dipolar interaction is expected to be

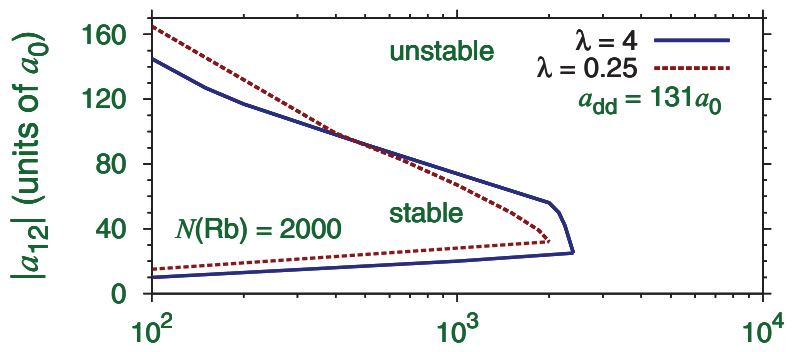

(a)

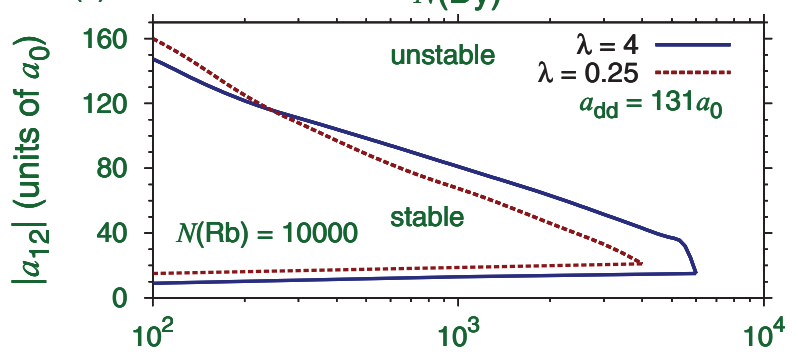

(b)

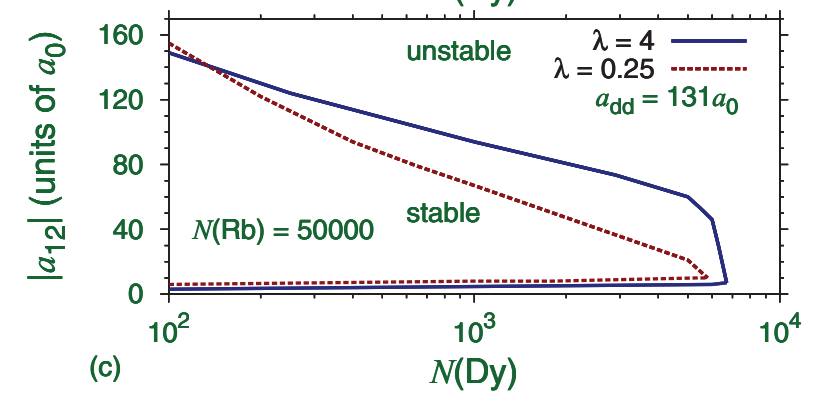

FIG. 1. (Color online) $\left|a_{12}\right|-N$ (Dy) stability plot showing the domain of stable ${ }^{164} \mathrm{Dy}$ droplets in a binary ${ }^{87} \mathrm{Rb}-{ }^{164} \mathrm{Dy}$ mixture for (a) $N(\mathrm{Rb})=2000$, (b) $N(\mathrm{Rb})=10000$, and (c) $N(\mathrm{Rb})=50000$, and for $\lambda=0.25$ and 4 . The intraspecies scattering lengths are taken as $a_{i}=110 a_{0}$.

more prominent, and not spherically symmetric ones where the effect of dipolar interaction is expected to be a minimum [1,5]. In all plots of Fig. 1, for a fixed $N(\mathrm{Rb})$, the ${ }^{164}$ Dy droplet can be bound for a maximum of $N(\mathrm{Dy})$. This maximum of $N$ (Dy) increases with $N(\mathrm{Rb})$, all other parameters remaining fixed. For a fixed $N(\mathrm{Rb})$, the disk-shaped trap can accommodate a larger maximum number of ${ }^{164} \mathrm{Dy}$ atoms than the cigar-shaped trap. For $N$ (Dy) smaller than this maximum number, the ${ }^{164}$ Dy droplet can be bound for $\left|a_{12}\right|$ between two limiting values. For $\left|a_{12}\right|$ above the upper limit, there is too much interspecies attraction on the the ${ }^{164}$ Dy droplet, leading to its collapse. For $\left|a_{12}\right|$ below the lower limit, there is not enough attraction and the ${ }^{164}$ Dy droplet cannot be bound. The lower limit of $\left|a_{12}\right|$ is small and tends to zero as $N(\mathrm{Rb})$ tends to infinity. For fixed values of $N(\mathrm{Rb})$ and $N(\mathrm{Dy})$ and for small $\left|a_{12}\right|$ near the lower limit, the disk-shaped configuration is favored, and it can bind the ${ }^{164}$ Dy droplet for smaller values of $\left|a_{12}\right|$. However, for a fixed $N(\mathrm{Rb})$ and for a large $\left|a_{12}\right|$ near the upper limit, the disk-shaped configuration is favored only for a large $N\left(\right.$ Dy) allowing a ${ }^{164}$ Dy droplet for larger $\left|a_{12}\right|$, whereas the cigar-shaped configuration is favored for a small $N(\mathrm{Dy})$ allowing a ${ }^{164}$ Dy droplet for larger $\left|a_{12}\right|$.

In Fig. 2 we illustrate the numerical and variational results for chemical potentials and root-mean-square (rms) sizes $\langle x\rangle$ 

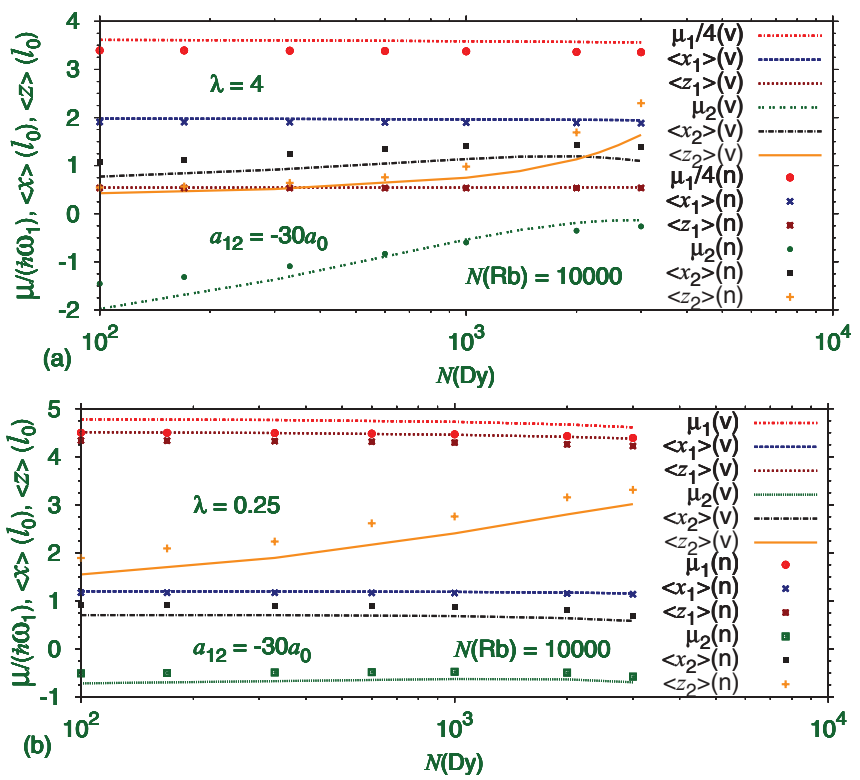

FIG. 2. (Color online) Variational (v) and numerical (n) results for chemical potential $\mu$ and rms sizes $\langle x\rangle$ and $\langle z\rangle$ for the trapped ${ }^{87} \mathrm{Rb}$ BEC of 10000 atoms and the bound ${ }^{164}$ Dy droplet versus the number of ${ }^{164} \mathrm{Dy}$ atoms for (a) $\lambda=4$ and (b) $\lambda=0.25$. The interspecies scattering length $a_{12}=-30 a_{0}$ and the subscript 1 refer to ${ }^{87} \mathrm{Rb}$ atoms and 2 to ${ }^{164}$ Dy atoms.

and $\langle z\rangle$ of the trapped ${ }^{87} \mathrm{Rb}$ BEC of 10000 atoms and of the bound ${ }^{164}$ Dy droplet versus $N$ (Dy) for (a) $\lambda=4$ and (b) 0.25 . Considering that the ${ }^{164}$ Dy droplet may not have a Gaussian shape as assumed in the variational approximation, the agreement between the numerical and variational results is quite satisfactory.

The existence of the quasifree ${ }^{164}$ Dy droplet can be studied qualitatively by a minimization of the energy (19). However, the widths of the ${ }^{87} \mathrm{Rb}$ BEC for a fixed $N(\mathrm{Rb})$ do not vary much as $N$ (Dy) or $a_{12}$ is varied. Hence, for a qualitative understanding of the existence of a stable ${ }^{164}$ Dy droplet for a fixed $N(\mathrm{Rb})$, we can make a further approximation in the energy (19) and take the widths of the trapped ${ }^{87} \mathrm{Rb} B E C$ to be the same as the widths of the ${ }^{87} \mathrm{Rb} \mathrm{BEC}$ in the absence of ${ }^{164} \mathrm{Dy}$ atoms under otherwise identical conditions. The widths of the ${ }^{164}$ Dy droplet so obtained for $N(\mathrm{Rb})=50000$ are compared in Table I for several values of $a_{12}, N$ (Dy), and $\lambda$ with the widths obtained from exact energy minimization. We find from Table

TABLE I. Root-mean-square sizes of the binary ${ }^{87} \mathrm{Rb}-{ }^{164} \mathrm{Dy}$ system of $50000{ }^{87} \mathrm{Rb}$ atoms from the variational approximation (11)-(14) (Var), and an approximate energy minimization (Approx).

\begin{tabular}{lccccccc}
\hline \hline & $a_{12}$ & $N($ Dy $)$ & $\lambda$ & $\left\langle x_{1}\right\rangle$ & $\left\langle z_{1}\right\rangle$ & $\left\langle x_{2}\right\rangle$ & $\left\langle z_{2}\right\rangle$ \\
\hline Approx & $-40 a_{0}$ & 1000 & 4 & 2.7597 & 0.7106 & 1.0755 & 0.4847 \\
Var & $-40 a_{0}$ & 1000 & 4 & 2.7475 & 0.7091 & 1.0703 & 0.4834 \\
Approx & $-80 a_{0}$ & 1000 & 0.25 & 2.2780 & 8.9525 & 0.6468 & 2.5799 \\
Var & $-80 a_{0}$ & 1000 & 0.25 & 2.2549 & 8.8558 & 0.6378 & 2.5525 \\
Approx & $-60 a_{0}$ & 500 & 4 & 2.7597 & 0.7106 & 0.8478 & 0.3768 \\
Var & $-60 a_{0}$ & 500 & 4 & 2.7492 & 0.7090 & 0.8443 & 0.3757 \\
Approx & $-100 a_{0}$ & 100 & 0.25 & 2.2780 & 8.9525 & 0.6384 & 1.5803 \\
Var & $-100 a_{0}$ & 100 & 0.25 & 2.2752 & 8.9392 & 0.6373 & 1.5779 \\
\hline \hline
\end{tabular}
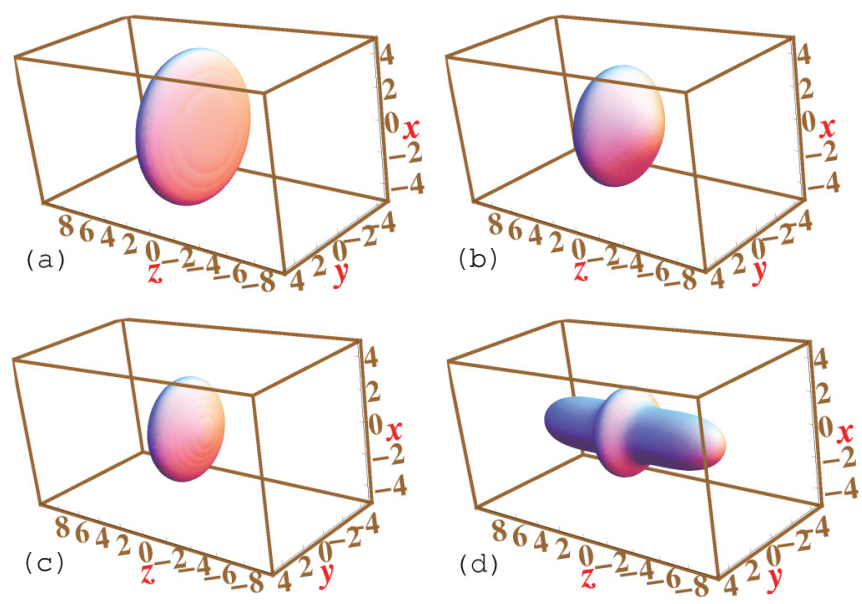

FIG. 3. (Color online) The isodensity contour of (a) a disk-shaped $(\lambda=4){ }^{87} \mathrm{Rb}$ BEC of 10000 atoms and that of the bound ${ }^{164} \mathrm{Dy}$ droplet of (b) 1000 atoms $\left(a_{12}=-30 a_{0}\right)$, (c) 1000 atoms $\left(a_{12}=-80 a_{0}\right)$, and (d) 4000 atoms $\left(a_{12}=-30 a_{0}\right)$. The isodensity contour of the ${ }^{87} \mathrm{Rb}$ BEC is practically the same in all three cases. The density on the contour in all cases is $0.001 l_{0}^{-3}$ and length is measured in units of $l_{0}$ $(=1 \mu \mathrm{m})$.

I that the size of the ${ }^{87} \mathrm{Rb}$ for a fixed $N(\mathrm{Rb})$ remains reasonably constant with respect to the variation of $N(\mathrm{Dy})$ and $a_{12}$ and that the approximate energy minimization with fixed widths for ${ }^{87} \mathrm{Rb}$ BEC leads to a good approximation to the widths of the ${ }^{164}$ Dy droplet. In the numerical solution of the binary GP equations (6) and (7), we also verified that the shape and size of the trapped ${ }^{87} \mathrm{Rb} \mathrm{BEC}$ are fairly independent of the presence or absence of the ${ }^{164}$ Dy droplet. Hence in the study of the shape, size, and dynamics of the dipolar ${ }^{164} \mathrm{Dy}$ droplet bound in the trapped ${ }^{87} \mathrm{Rb} \mathrm{BEC}$, the trapped BEC will have only a passive role and we shall highlight only the shape, size, and dynamics of the ${ }^{164}$ Dy droplet in the following.

We consider the shape of a stable ${ }^{164}$ Dy droplet in the stability plots of Fig. 1 and study its change as the collapse instability is approached. In Fig. 3(a) we show the isodensity contour plot [density $\left|\phi_{1}(\mathbf{r})\right|^{2}$ ] of a disk-shaped $(\lambda=4){ }^{87} \mathrm{Rb}$ BEC of 10000 atoms and that [density $\left.\left|\phi_{2}(\mathbf{r})\right|^{2}\right]$ of the bound ${ }^{164}$ Dy droplet in Fig. 3(b) for $N($ Dy $)=1000\left(a_{12}=-30 a_{0}\right)$, in Fig. 3(c) for $N(\mathrm{Dy})=1000\left(a_{12}=-80 a_{0}\right)$, and in Fig. 3(d) for $N($ Dy $)=4000\left(a_{12}=-30 a_{0}\right)$. The isodensity of the ${ }^{87} \mathrm{Rb}$ BEC is practically the same in all three cases. Of the isodensities of the ${ }^{164}$ Dy droplet, the one in Fig. 3(b) is deep inside the stability region far away from collapse instability. The parameters of Figs. 3(c) and 3(d) are close to the region of collapse instability. Of these two, Fig. 3(c) corresponds to a medium number of ${ }^{164}$ Dy atoms and Fig. 3(d) to a large number. Independent of the initial shape, a ${ }^{164}$ Dy droplet with a medium number of ${ }^{164} \mathrm{Dy}$ atoms always collapses towards the center with shrinking size. However, a ${ }^{164}$ Dy droplet containing a large number of atoms, independent of the associated trap symmetry, always first takes a cigar shape and then collapses on the axial $z$ direction. A strong dipolar interaction prohibits a collapse to center of a large ${ }^{164}$ Dy droplet and favors a cigar shape. In Fig. 3 the trap is disk shaped. The medium-sized ${ }^{164}$ Dy droplet of Fig. 3(c) collapses to center, maintaining the disk shape, as the net dipolar interaction is smaller in this case. The large ${ }^{164} \mathrm{Dy}$ 

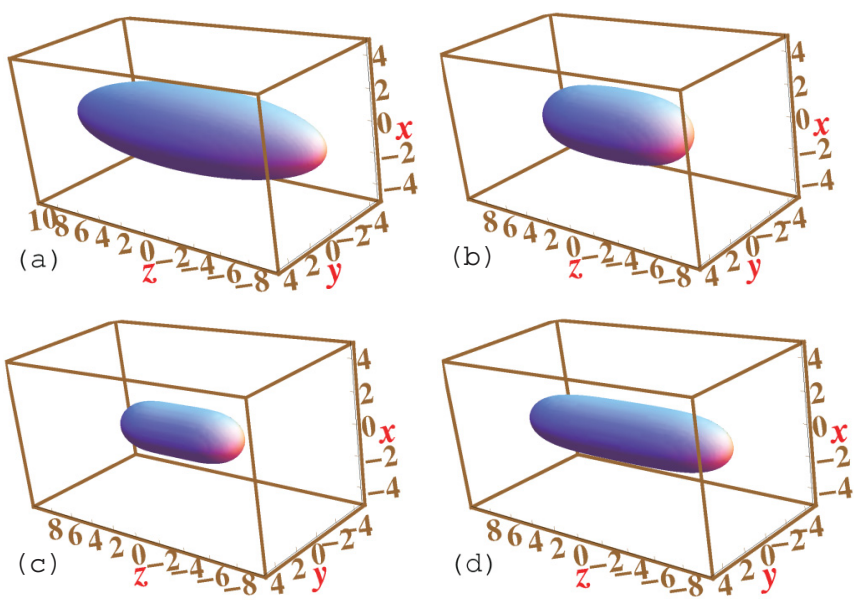

FIG. 4. (Color online) The isodensity contour of (a) a cigarshaped $(\lambda=0.25){ }^{87} \mathrm{Rb} \mathrm{BEC}$ of 10000 atoms and that of the bound ${ }^{164}$ Dy droplet of (b) 1000 atoms $\left(a_{12}=-30 a_{0}\right)$, (c) 1000 atoms $\left(a_{12}=-65 a_{0}\right)$, and (d) 3000 atoms $\left(a_{12}=-30 a_{0}\right)$. The isodensity contour of the ${ }^{87} \mathrm{Rb} \mathrm{BEC}$ is practically the same in all three cases. The density on the contour in all cases is $0.001 l_{0}^{-3}$ and length is measured in units of $l_{0}(=1 \mu \mathrm{m})$.

droplet in Fig. 3(d) has changed its shape from the supporting disk-shaped trap to a cigar shape. If the attraction is further increased by increasing $\left|a_{12}\right|$, the ${ }^{164}$ Dy droplet of Fig. 3(d) would collapse from the cigar shape on the axial $z$ axis.

In Fig. 4(a) we illustrate the isodensity contour of a cigarshaped $(\lambda=0.25){ }^{87} \mathrm{Rb}$ BEC of 10000 atoms, and the same for the ${ }^{164}$ Dy droplet are shown in Fig. 4(b) for $N(\mathrm{Dy})=1000$ $\left(a_{12}=-30 a_{0}\right)$, in Fig. 4(c) for $N(\mathrm{Dy})=1000\left(a_{12}=-65 a_{0}\right)$, and in Fig. 4(d) for $N(\mathrm{Dy})=3000\left(a_{12}=-30 a_{0}\right)$. The density of the ${ }^{87} \mathrm{Rb} \mathrm{BEC}$ is virtually the same in all three cases. Of the three ${ }^{164}$ Dy droplets, the one in Fig. 4(b) is deep inside the stability region far away from collapse instability. The parameters for Figs. 4(c) and 4(d) are close to the region of collapse instability. In all cases the ${ }^{164}$ Dy droplet maintains the cigar shape of the accompanying trap acting on the ${ }^{87} \mathrm{Rb}$ BEC. The size of the ${ }^{164}$ Dy droplet in Fig. 4(b) is larger than that of Fig. 4(c) with the same number of atoms due to a strong interspecies attraction acting on the latter. The ${ }^{164}$ Dy droplet of Fig. 4(d) is larger than those in Figs. 4(b) and 4(c) due to the larger number of ${ }^{164} \mathrm{Dy}$ atoms in it.

We investigate the dynamics of a bound ${ }^{164}$ Dy droplet in a trapped ${ }^{87} \mathrm{Rb}$ BEC. First, to test the present scheme we consider a small ${ }^{164} \mathrm{Dy}$ droplet of 100 atoms bound in a disk-shaped $(\lambda=4){ }^{87} \mathrm{Rb}$ BEC of 2000 atoms with $a_{12}=-50 a_{0}$. This corresponds to the stable region of Fig. 1(a). In real-time evolution of the system, the scattering length $a_{12}$ is suddenly changed to $1.01 a_{12}$, thus starting the breathing oscillation. In Fig. 5 we show the resultant oscillation of the rms sizes (a) $\langle\rho\rangle$ and (b) $\langle r\rangle$ during time evolution as obtained from numerical solution of Eqs. (6) and (7) and the variational approximation (11)-(14).

After obtaining a satisfactory result for the dynamics with a small system, we venture with a larger system of experimental interest, e.g., a stable ${ }^{164}$ Dy droplet of 1000 atoms bound in a cigar-shaped $(\lambda=0.25){ }^{87} \mathrm{Rb}$ BEC of 10000 atoms with $a_{12}=-50 a_{0}$ corresponding to the stable region of Fig. 1(b).
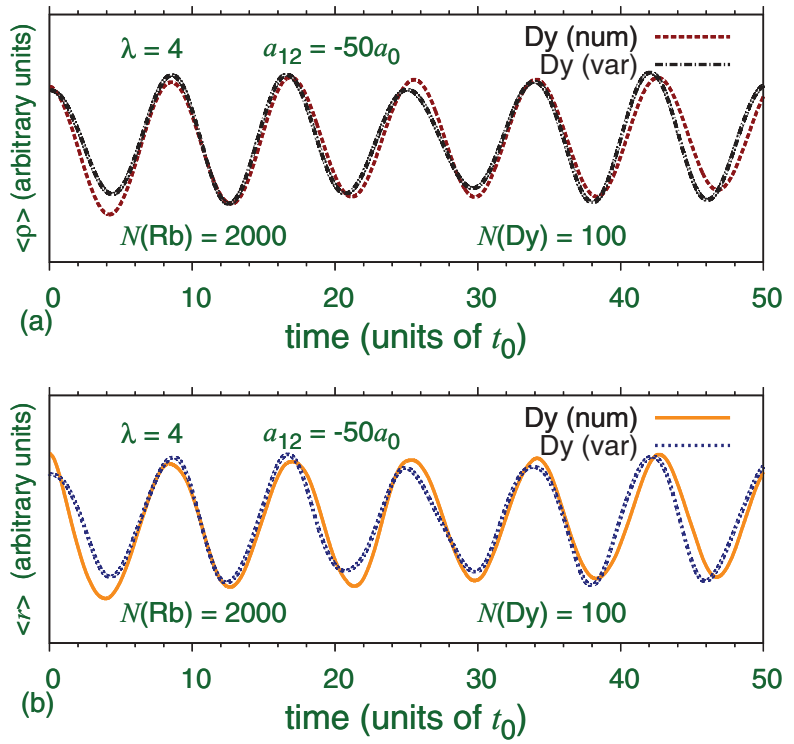

FIG. 5. (Color online) The rms sizes (a) $\langle\rho\rangle$ and (b) $\langle r\rangle$ from numerical simulation (num) and variational approximation (var) during breathing oscillation initiated by the sudden change $a_{12} \rightarrow$ $1.01 a_{12}$ versus time in units of $t_{0}(=1.38 \mathrm{~ms})$ of a ${ }^{164} \mathrm{Dy}$ droplet of 100 atoms bound in a trapped ${ }^{87} \mathrm{Rb}$ BEC of 2000 atoms for $a_{12}=-50$ and $\lambda=4$.

In real-time evolution of the system, the scattering length $a_{12}$ is again suddenly changed to $1.01 a_{12}$, thus starting the breathing oscillation. In Fig. 6 we show the resultant oscillation of the rms sizes (a) $\langle z\rangle$ and (b) $\langle x\rangle$ during time evolution as obtained from a numerical solution of Eqs. (6) and (7) and the variational approximation (11)-(14). The agreement between the numerical simulation and variational approximation in
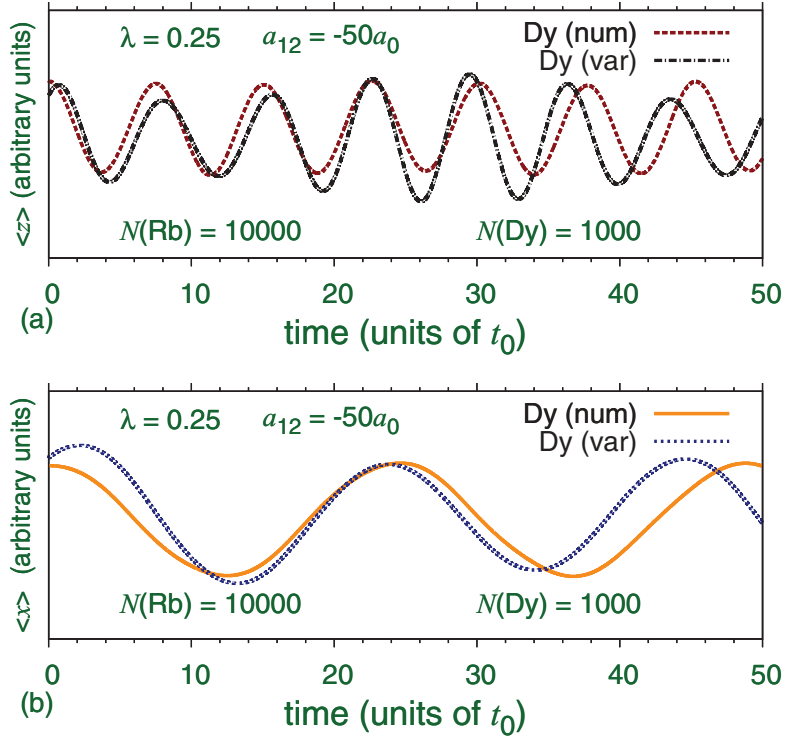

FIG. 6. (Color online) The rms sizes (a) $\langle z\rangle$ and (b) $\langle x\rangle$ from numerical simulation (num) and variational approximation (var) during breathing oscillation initiated by the sudden change $a_{12} \rightarrow$ $1.01 a_{12}$ versus time in units of $t_{0}(=1.38 \mathrm{~ms})$ of a ${ }^{164} \mathrm{Dy}$ droplet of 1000 atoms bound in a trapped ${ }^{87} \mathrm{Rb}$ BEC of 10000 atoms for $a_{12}=-50$ and $\lambda=0.25$. 
both cases of breathing oscillation illustrated in Figs. 5 and 6 for disk and cigar shapes is quite satisfactory considering the facts that (i) during oscillation the bound ${ }^{164}$ Dy droplet might have a density distribution different from the Gaussian distribution assumed in the variational approximation and (ii) the large nonlinearity in the ${ }^{87} \mathrm{Rb} \mathrm{BEC}$ will also make its density distribution non-Gaussian. The stable oscillation under small perturbation as shown in Figs. 5 and 6 confirms the dynamical stability of the ${ }^{164}$ Dy droplet for both disk- and cigar-type environments.

The present quasifree dipolar droplet is not just of theoretical interest, but can be realized experimentally by initially preparing a binary ${ }^{87} \mathrm{Rb}-{ }^{164} \mathrm{Dy}$ mixture where both the components are harmonically trapped. The trap on ${ }^{164} \mathrm{Dy}$ can then be ramped to zero exponentially during a few milliseconds. The ${ }^{164}$ Dy cloud will initially expand and finally form the quasifree ${ }^{164} \mathrm{Dy}$ droplet. To illustrate numerically the viability of this procedure, we consider an initial ${ }^{87} \mathrm{Rb}-{ }^{164} \mathrm{Dy}$ binary mixture with the following parameters: $N(\mathrm{Rb})=$ $10000, N(\mathrm{Dy})=1000$, and $a_{12}=-50 a_{0}$. We take the same axial trap with $\lambda=4$ acting on both components. Then we perform real-time propagation of the binary GP equation with this initial state and ramp down the ${ }^{164}$ Dy trap by $V_{\text {trap }}(\mathrm{Dy}) \rightarrow \exp [-(t-10)] V_{\text {trap }}(\mathrm{Dy})$ for time $t>10$. The trap on ${ }^{164} \mathrm{Dy}$ is practically zero for times $t \geqslant 20$. The trapped ${ }^{164}$ Dy BEC first expands for $t>10$ and eventually it emerges as the quasifree ${ }^{164}$ Dy droplet. The time evolution of the rms sizes of the ${ }^{164}$ Dy droplet is shown in Fig. 7(a). The widths of the ${ }^{164}$ Dy droplet first increase and eventually execute small
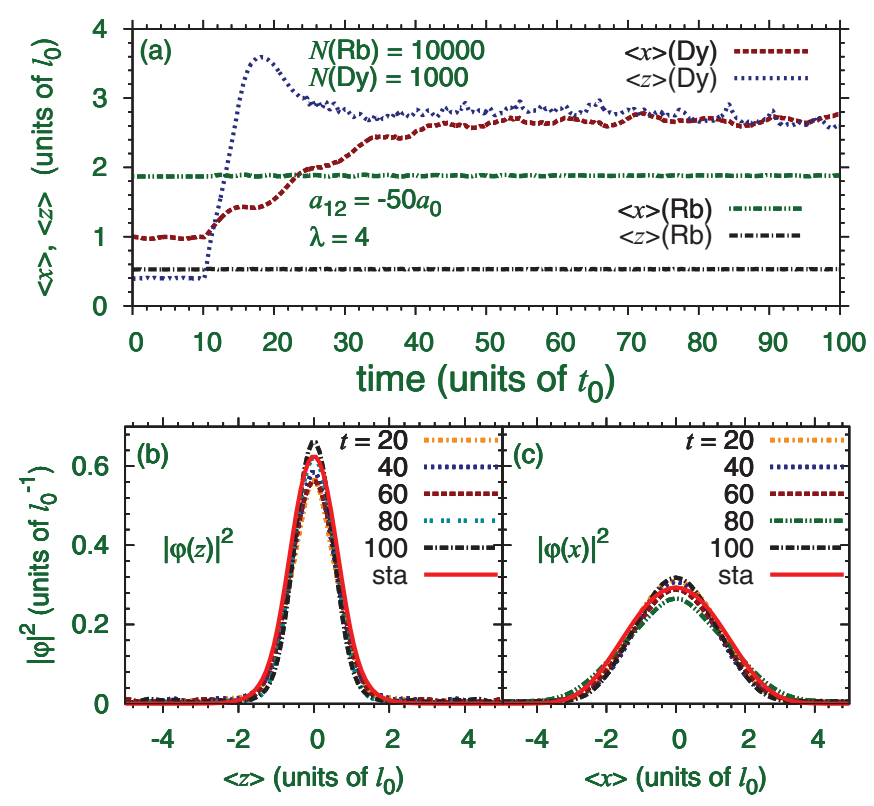

FIG. 7. (Color online) (a) The rms sizes $\langle x\rangle$ and $\langle z\rangle$ of the ${ }^{164} \mathrm{Dy}$ and ${ }^{87} \mathrm{Rb} \mathrm{BECs}$ versus time during the passage of the trapped ${ }^{164} \mathrm{Dy}$ BEC in the binary ${ }^{87} \mathrm{Rb}-{ }^{164} \mathrm{Dy}$ mixture with $N(\mathrm{Rb})=10000$, $N(\mathrm{Dy})=1000, a_{12}=-50 a_{0}$, and $\lambda=4$ as the confining trap on the ${ }^{164}$ Dy BEC is relaxed for $t>10$ exponentially as $V_{\text {trap }} \rightarrow \exp [-(t-$ 10)] $V_{\text {trap }}$, so that this trap is practically zero for $t>20$. The linear 1 D densities (b) $|\varphi(z)|^{2}$ and (c) $|\varphi(x)|^{2}$ at times $t=20,40,60,80,100$ (in units of $t_{0}$ ) during the expansion together with the numerically calculated density of the stationary (sta) ${ }^{164}$ Dy droplet. oscillations illustrating the stable ${ }^{164}$ Dy droplet. To investigate the shape of the ${ }^{164}$ Dy droplet, we plot in Figs. 7(b) and 7(c) the one-dimensional densities $|\varphi(z)|^{2} \equiv \iint d x d y|\phi(x, y, z)|^{2}$ and $|\varphi(x)|^{2} \equiv \iint d y d z|\phi(x, y, z)|^{2}$ at times $t=20,40,60,80$, and 100 along with the corresponding numerical densities of the stationary droplet calculated from the solution of Eqs. (6) and (7). The $1 \mathrm{D}$ numerical densities of the stationary ${ }^{164} \mathrm{Dy}$ droplet are in agreement with the densities obtained from dynamical simulation of the passage of the trapped ${ }^{164} \mathrm{Dy}$ BEC to a quasifree droplet. In Figs. 7(b) and 7(c) one can easily identify the small oscillation of the evolving dynamical droplet around its stationary shape.

\section{SUMMARY AND DISCUSSION}

Using variational approximation and numerical solution of a set of coupled mean-field GP equations, we demonstrate the existence of a stable dipolar ${ }^{164}$ Dy droplet bound by interspecies attraction in a trapped nondipolar BEC of ${ }^{87} \mathrm{Rb}$ atoms. The domain of stability of the ${ }^{164} \mathrm{Dy}$ droplet is highlighted in stability plots of the number of ${ }^{164} \mathrm{Dy}$ atoms and interspecies scattering length $a_{12}$ for both cigar- and diskshaped traps acting on the ${ }^{87} \mathrm{Rb} \mathrm{BEC}$. Results of the variational approximation and numerical solution for the statics (sizes and chemical potentials) and dynamics (breathing oscillation) of the ${ }^{164}$ Dy droplet are found to be in satisfactory agreement with each other. We also demonstrate numerically that such droplets can be obtained experimentally by considering a trapped binary ${ }^{87} \mathrm{Rb}-{ }^{164} \mathrm{Dy}$ BEC and then removing the trap on ${ }^{164}$ Dy. The ${ }^{164}$ Dy BEC then expands and transforms into a bound dipolar droplet.

Dipolar interaction among atoms is quite different from the normal short-range atomic interaction and manifests itself in different ways in a trapped dipolar BEC. However, in a trapped dipolar BEC, the confining constraints could be too strong and could make the effect of dipolar interaction difficult to observe. A special experimental set up and theoretical formulation might be necessary to study the effect of dipolar interaction. However, it will be much easier to see the effect of dipolar interaction in the present binary mixture of nondipolar ${ }^{87} \mathrm{Rb}$ and dipolar ${ }^{164} \mathrm{Dy}$. Strong dipolar interaction in the axial polarization $z$ direction should elongate the dipolar BEC along this direction, thus transforming it to a cigar shape. However, it will be difficult to observe this change in the presence of a harmonic trap along $z$. The effect of dipolar interaction is clearly seen in the present quasifree ${ }^{164}$ Dy droplet of Fig. 3(d), where due to a strong dipolar interaction the shape of the ${ }^{164} \mathrm{Dy}$ droplet has changed from the disk shape-the shape of the ${ }^{87} \mathrm{Rb}$ BEC of Fig. 3(a) responsible for its binding-to the cigar shape. The thin disk-shaped ${ }^{87} \mathrm{Rb}$ BEC stays only near the central $z=0$ plane of the ${ }^{164} \mathrm{Dy}$ droplet. Most of the ${ }^{164} \mathrm{Dy}$ atoms lying outside the ${ }^{87} \mathrm{Rb}$ BEC are bound due to the intraspecies dipolar interaction. In the dipolar ${ }^{164}$ Dy droplet of Fig. 3(d) the dipolar interaction is playing a more important role in its binding and shape.

The present quasifree dipolar droplet may also find other interesting applications in the BEC phenomenology. Among the interesting features in a trapped dipolar BEC, one can mention its peculiar shape and stability properties [11], $D$ wave collapse [19], anisotropic solitons, vortex solitons [20] 
and vortex lattices [21], anisotropic shock- and sound-wave propagation [22], anisotropic Landau critical velocity [23], stable checkerboard, stripe, and star configurations in a twodimensional optical lattice such as a stable Mott insulator [24], as well as superfluid soliton [25] states. It would be of great interest to find out how these features and properties of a trapped dipolar BEC would manifest in a 3D quasifree dipolar droplet. For example, a quasifree dipolar droplet could be used in the experimental study of anisotropic sound- and shock-wave propagation [22], collapse dynamics [19], anisotropic Landau critical velocity [23], formation of vortex dipoles and vortex lattices [21], etc., in a different setting of confinement which will facilitate the observation of the effect of the anisotropic dipolar interaction.

\section{ACKNOWLEDGMENTS}

We thank FAPESP and CNPq (Brazil) for partial support.
[1] T. Lahaye, C. Menotti, L. Santos, M. Lewenstein, and T. Pfau, Rep. Prog. Phys. 72, 126401 (2009).

[2] T. Lahaye, T. Koch, B. Fröhlich, M. Fattori, J. Metz, A. Griesmaier, S. Giovanazzi, and T. Pfau, Nature (London) 448, 672 (2007); A. Griesmaier, J. Stuhler, T. Koch, M. Fattori, T. Pfau, and S. Giovanazzi, Phys. Rev. Lett. 97, 250402 (2006).

[3] J. Stuhler, A. Griesmaier, T. Koch, M. Fattori, T. Pfau, S. Giovanazzi, P. Pedri, and L. Santos, Phys. Rev. Lett. 95, 150406 (2005).

[4] K. Goral, K. Rzazewski, and T. Pfau, Phys. Rev. A 61, 051601 (2000).

[5] T. Koch et al., Nat. Phys. 4, 218 (2008).

[6] M. Lu, S. H. Youn, and B. L. Lev, Phys. Rev. Lett. 104, 063001 (2010); J. J. McClelland and J. L. Hanssen, ibid. 96, 143005 (2006); S. H. Youn, M. W. Lu, U. Ray, and B. V. Lev, Phys. Rev. A 82, 043425 (2010).

[7] M. Lu, N. Q. Burdick, Seo Ho Youn, and B. L. Lev, Phys. Rev. Lett. 107, 190401 (2011).

[8] K. Aikawa, A. Frisch, M. Mark, S. Baier, A. Rietzler, R. Grimm, and F. Ferlaino, Phys. Rev. Lett. 108, 210401 (2012).

[9] J. Deiglmayr, A. Grochola, M. Repp, K. Mortlbauer, C. Gluck, J. Lange, O. Dulieu, R. Wester, and M. Weidemuller, Phys. Rev. Lett. 101, 133004 (2008); M. H. G. de Miranda, A. Chotia, B. Neyenhuis, D. Wang, G. Quéméner, S. Ospelkaus, J. L. Bohn, J. Ye, and D. S. Jin, Nat. Phys. 7, 502 (2011); J. Doyle, B. Friedrich, R. V. Krems, and F. Masnou-Seeuws, Eur. Phys. J. D 31, 149 (2004).

[10] F. Dalfovo, S. Giorgini, L. P. Pitaevskii, and S. Stringari, Rev. Mod. Phys. 71, 463 (1999).

[11] N. G. Parker, C. Ticknor, A. M. Martin, and D. H. J. O'Dell, Phys. Rev. A 79, 013617 (2009); R. M. Wilson, S. Ronen, and J. L. Bohn, ibid. 80, 023614 (2009); N. G. Parker and D. H. J. O’Dell, ibid. 78, 041601 (2008); L. Santos, G. V. Shlyapnikov, P. Zoller, and M. Lewenstein, Phys. Rev. Lett. 85, 1791 (2000); C. Ticknor, N. G. Parker, A. Melatos, S. L. Cornish, D. H. J. O'Dell, and A. M. Martin, Phys. Rev. A 78, 061607 (2008); R. M. W. van Bijnen, A. J. Dow, D. H. J. O’Dell, N. G. Parker, and A. M. Martin, ibid. 80, 033617 (2009); A. Junginger, J. Main, G. Wunner, and T. Bartsch, ibid. 86, 023632 (2012); M. Asad-uz-Zaman and D. Blume, ibid. 80, 053622 (2009);
S. Ronen, D. C. E. Bortolotti, and J. L. Bohn, Phys. Rev. Lett. 98, 030406 (2007).

[12] R. M. Wilson, C. Ticknor, J. L. Bohn, and E. Timmermans, Phys. Rev. A 86, 033606 (2012); H. Saito, Y. Kawaguchi, and M. Ueda, Phys. Rev. Lett. 102, 230403 (2009).

[13] Luis E. Young-S. and S. K. Adhikari, Phys. Rev. A 86, 063611 (2012).

[14] S. Yi and L. You, Phys. Rev. A 63, 053607 (2001).

[15] K. Goral and L. Santos, Phys. Rev. A. 66, 023613 (2002).

[16] V. M. Perez-Garcia, H. Michinel, J. I. Cirac, M. Lewenstein, and P. Zoller, Phys. Rev. A 56, 1424 (1997).

[17] P. Muruganandam and S. K. Adhikari, Comput. Phys. Commun. 180, 1888 (2009); D. Vudragovic, I. Vidanovic, A. Balaz, P. Muruganandam, and S. K. Adhikari, ibid. 183, 2021 (2012).

[18] S. Inouye et al., Nature (London) 392, 151 (1998).

[19] T. Lahaye, J. Metz, B. Frohlich, T. Koch, M. Meister, A. Griesmaier, T. Pfau, H. Saito, Y. Kawaguchi, and M. Ueda, Phys. Rev. Lett. 101, 080401 (2008).

[20] I. Tikhonenkov, B. A. Malomed, and A. Vardi, Phys. Rev. Lett. 100, 090406 (2008); S. K. Adhikari and P. Muruganandam, J. Phys. B 45, 045301 (2012); Luis E. Young-S., P. Muruganandam, and S. K. Adhikari, ibid. 44, 101001 (2011); P. Muruganandam and S. K. Adhikari, ibid. 44, 121001 (2011); P. Köberle, D. Zajec, G. Wunner, and B. A. Malomed, Phys. Rev. A 85, 023630 (2012).

[21] R. M. W. van Bijnen, D. H. J. O’Dell, N. G. Parker, and A. M. Martin, Phys. Rev. Lett. 98, 150401 (2007); R. Kishor Kumar and P. Muruganandam, J. Phys. B 45, 215301 (2012); M. Abad, M. Guilleumas, R. Mayol, M. Pi, and D. M. Jezek, Phys. Rev. A 79, 063622 (2009).

[22] P. Muruganandam and S. K. Adhikari, Phys. Lett. A 376, 480 (2012); C. Krumnow and A. Pelster, Phys. Rev. A 84, 021608 (2011).

[23] R. M. Wilson, S. Ronen, and J. L. Bohn, Phys. Rev. Lett. 104, 094501 (2010).

[24] B. Capogrosso-Sansone, C. Trefzger, M. Lewenstein, P. Zoller, and G. Pupillo, Phys. Rev. Lett. 104, 125301 (2010).

[25] K. Lakomy, R. Nath, and L. Santos, Phys. Rev. A 85, 033618 (2012); S. K. Adhikari and P. Muruganandam, Phys. Lett. A 376, 2200 (2012). 